\title{
Update of aristolochic acid nephropathy in Korea
}

Tae Hyun Ban ${ }^{1}$, Ji-Won Min ${ }^{1}$, Changhwan $\mathrm{Seo}^{2}$, Da Rae Kim³ , Yu Ho Lee ${ }^{3}$, Byung Ha Chung ${ }^{1}$, Kyung-Hwan Jeong 3 , Jae Wook Lee ${ }^{4}$, Beom Seok Kim², Sang-Ho Lee ${ }^{3}$, Bum Soon Choi ${ }^{1}$, Jin Suk Han ${ }^{5}$, and Chul Woo Yang ${ }^{1}$

\begin{abstract}
${ }^{1}$ Division of Nephrology, Department of Internal Medicine, College of Medicine, The Catholic University of Korea, Seoul; ${ }^{2}$ Department of Internal Medicine, The Research Institute for Transplantation, and Institute of Kidney Disease Research, Yonsei University College of Medicine, Seoul; ${ }^{3}$ Division of Nephrology, Department of Internal Medicine, Kyung Hee University College of Medicine, Seoul; ${ }^{4}$ Nephrology Clinic, National Cancer Center Hospital, Goyang; ${ }^{5}$ Department of Internal Medicine, Seoul National University College of Medicine, Seoul, Korea
\end{abstract}

Received: September 26, 2016 Revised : October 31, 2016 Accepted: November 8, 2016

\section{Correspondence to}

Chul Woo Yang, M.D.

Department of Internal Medicine, College of Medicine, Seoul St. Mary's Hospital, The Catholic University of Korea,

222 Banpo-daero, Seocho-gu,

Seoul o6591, Korea

Tel: +82-2-2258-6037

Fax: +82-2-536-0323

E-mail: yangch@catholic.ac.kr
Background/Aims: The true incidence of aristolochic acid nephropathy (AAN) is thought to be underestimated because numerous ingredients known or suspected to contain aristolochic acid (AA) are used in traditional medicine in Korea.

Methods: We collected data on cases of AAN since 1996 via a database in Korea. We evaluated the year of AAN development, route to obtaining AA-containing herbal medicine, gender, reason for taking AA-containing herbal medicine, clinical manifestations, histological findings, phytochemical analysis, and prognosis of patients with AAN.

Results: Data on 16 cases of AAN were collected. Thirteen cases developed AAN before and three cases after the prohibition of AA-containing herbal medicine by the Korea Food and Drug Administration. Patients were prescribed AA-containing herbal medicine from oriental clinics or had purchased it from traditional markets. AAN was distributed in all age groups. Young females were most commonly exposed to AA-containing herbal medicine for slimming purposes and postpartum health promotion, while older adults took AA-containing compounds for the treatment of chronic diseases. The most common symptoms presented at hospitalization were nausea and vomiting, and acute kidney injury was accompanied by Fanconi syndrome in almost half of the patients. Phytochemical analysis of AA in herbal medicine was available in six cases. Progression to end stage renal disease (ESRD) was observed in seven patients (43.8\%), and five patients (31.3\%) had progressed to ESRD within 6 months of diagnosis.

Conclusions: Our report shows that patients were still exposed to AA-containing herbal medicine and that there is a possibility of underdiagnosis of AAN in Korea. A stronger national supervision system of herbal ingredients and remedies in oriental medicine is needed to prevent AAN.

Keywords: Chinese herbal medicine; Aristolochic acid; Renal toxicity

\section{INTRODUCTION}

Aristolochic acid (AA)-containing compounds have been used as antiviral, antibacterial, anti-inflammatory, anti-neoplastic, and slimming agents $[1,2]$ for a long time. However, these compounds have become notorious after the breakout of interstitial nephritis in Europe- an women who took these compounds to reduce body weight [3]. The first case of aristolochic acid nephropathy (AAN) was reported in Belgium [3], and several cases with irreversible renal toxicity after intake of AA-containing compounds have been reported. More recently, AA has also been identified as a carcinogen responsible for urothelial carcinoma [2,4-6]. Accordingly, the World 
Health Organization classified AA as a type 1 carcinogen [2].

Due to both the renal toxicity and potential carcinogenic effects of AA, the Food and Drug Administration of the United States advised caution regarding the consumption of AA-containing compounds in 2000. In Korea, the Korea Food and Drug Administration (KFDA) prohibited herbal ingredients containing AA in 2005. However, even after AA-containing ingredients were prohibited in many countries, cases identified as AAN have been sporadically reported [3,7-15].

A wide range of plants contains AA [1]. Therefore, AA-containing compounds can still be acquired in traditional medicine clinics and through internet markets even though many countries have designated AA-containing compounds as prohibited substances $[1,16]$. Therefore, it is expected that more cases of AAN have developed in Korea. Herein, we collected cases of AAN by searching past publications in Korea and summarized a total of 16 cases with a review of the literature.

\section{METHODS}

\section{Study population}

We searched the Research Information Service System, Korean Medical Database, and the Korean Studies Information Service System using the phrases "Chinese herb nephropathy (CHN)" or "AAN." We reviewed the medical records of all patients with CHN or AAN [11,17-
22]. We also reviewed the medical records of patients proven to have AAN in four hospitals from January 2002 to December 2015. This study was approved by the Institutional Review Board of Seoul St. Mary's Hospital (IRB No. KC16RISIo735) and the Institutional Review Boards of each participating hospital. The informed consent was waived.

\section{Clinical parameters}

From the medical records, we obtained clinical information, including age, gender, past medical history, initial symptoms and physical examination, treatment, and clinical course. We also collected hematological and biochemical parameters and histopathologic findings, including hemoglobin, blood urea nitrogen (BUN), creatinine, AA level by phytochemical analysis, urinalysis, and histopathologic findings of the kidney.

\section{Phytochemical analysis and renal biopsy}

For the phytochemical analysis, we were able to obtain the remaining herbal medication from six of the patients diagnosed with AAN. The procured medical ingredients were analyzed by HPLC (high-performance liquid chromatography), and the results were expressed as $\mathrm{mg} / \mathrm{kg}$. AA is a mixture of structurally related nitrophenantrene carboxylic acids that consist of several subtypes, mainly 8-methoxy-6-nitro-phenanthro-(3,4-d)-1,3-dioxolo-5-carboxylic acid (AAI) and 6-nitro-phenanthro-(3,4-d)-1,3-dioxolo-5-carboxylic acid (AAII). Renal biopsy was conducted in all patients at first hospitalization. Renal tissue was

Table 1. Suggested diagnostic criteria for aristolochic acid nephropathy

\begin{tabular}{|c|c|}
\hline \multirow[t]{5}{*}{ Definitive } & Impaired renal function $\left(\mathrm{eGFR}<60 \mathrm{~mL} / \mathrm{min} / 1.73 \mathrm{~m}^{2}\right)$ plus \\
\hline & Any two of the following three criteria \\
\hline & 1. Characteristic renal histopathology ${ }^{\mathrm{a}}$ \\
\hline & 2. Ingestion of products containing AA confirmed by phytochemical analysis \\
\hline & 3. Presence of AA-DNA adducts in renal or urinary tract tissue \\
\hline \multirow[t]{3}{*}{ Probable } & Impaired renal function $\left(\mathrm{eGFR}<60 \mathrm{~mL} / \mathrm{min} / 1.73 \mathrm{~m}^{2}\right)$ plus \\
\hline & Only one of the above three criteria plus \\
\hline & Urothelial cancer at the time of presentation \\
\hline \multirow[t]{3}{*}{ Possible } & Impaired renal function $\left(\mathrm{eGFR}<60 \mathrm{~mL} / \mathrm{min} / 1.73 \mathrm{~m}^{2}\right)$ plus \\
\hline & No alternative explanation for renal dysfunction plus \\
\hline & History of use of herbal remedy likely to contain AA but without phytochemical confirmation \\
\hline
\end{tabular}

eGFR, estimated glomerular filtration rate; AA, aristolochic acid.

${ }^{a}$ Hypocellular interstitial fibrosis associated with tubular atrophy decreasing from the outer to the inner renal cortex. 
prepared for observation with light microscopy using hematoxylin and eosin, periodic acid-Schiff reaction, and silver methenamine and trichrome staining, and was also investigated via electron microscopy and immunofluorescence staining.

\section{Diagnosis of AAN}

We diagnosed AAN based on the diagnostic criteria of AAN suggested by Gokmen et al. (Table 1) [6]. The details included impaired renal function, renal histopathology, confirmation of AA by phytochemical analysis, and proof of AA-DNA adducts in renal or urinary tract tissue. If unexplained acute kidney injury (AKI) was accompanied by characteristic renal histology findings (tubulointerstitial nephritis, interstitial fibrosis, and tubular atrophy with preserved glomeruli by light microscopy without other abnormalities), it was considered to be compatible with the histopathology of AAN. Depending on the compatibility of our findings with the diagnostic criteria, patients were divided into definitive, probable, and possible AAN groups.

\section{Statistical analysis}

Statistical analyses were performed using SPSS version 18.0 (SPSS Inc., Chicago, IL, USA). Data were presented as the mean \pm standard deviation or the count and pere centage to the nearest tenth. Chi-square analysis was used for comparisons between the two groups, and a $p$ value less than 0.05 was considered statistically significant.

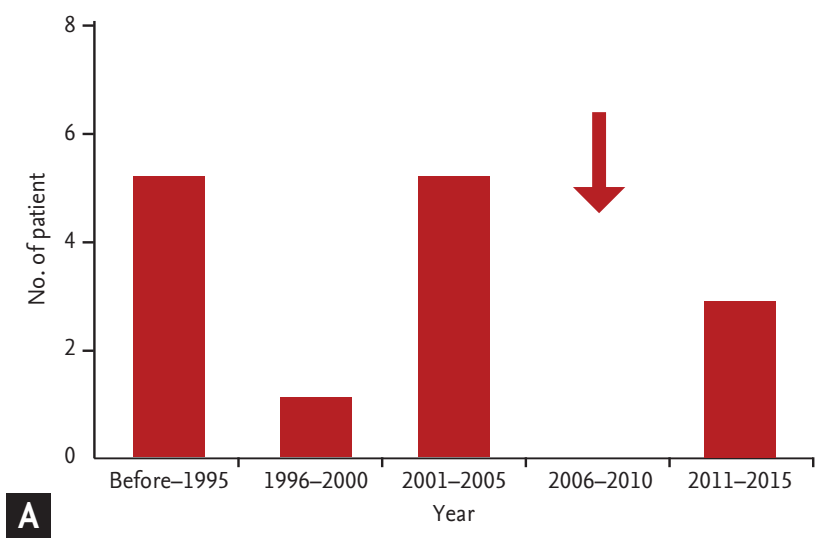

\section{RESULTS}

\section{Classification of patients based on diagnostic criteria} All cases satisfied the diagnostic criteria for AAN. Of the 16 cases, six (37.5\%) were classified as definite AAN and $10(62.5 \%)$ were classified as possible AAN. There were no cases of probable AAN.

\section{Occurrence and route of obtaining AA-containing drugs}

Fig. 1A shows the occurrence of AAN over a period of 20 years in Korea. When we divided the occurrence of AAN by every 5 years since 1996, 13 patients (81.3\%) had developed AAN before 2005, which is the year the KFDA prohibited AA-containing ingredients in herbal medicine. After prohibition, there was no occurrence of AAN between 2006 and 2010. However, three cases of AAN developed between 2011 and 2015. This finding clearly shows that the KFDA probation on the use of AA-containing ingredients in herbal medicine is not sufficient to prevent the occurrence of AAN in Korea. With respect to the route of obtaining AA-containing remedies, 11 cases (68.7\%) had received prescriptions from official oriental clinics, and five patients (31.3\%) had purchased herbal medicine from a traditional market (Fig. 1B). This finding shows that patients have easy access to AA-containing herbal medicine.

\section{Basic characteristics of patients}

Thirteen patients $(81.3 \%)$ were originally healthy, and three patients $(18.7 \%)$ had past history of medical dis-

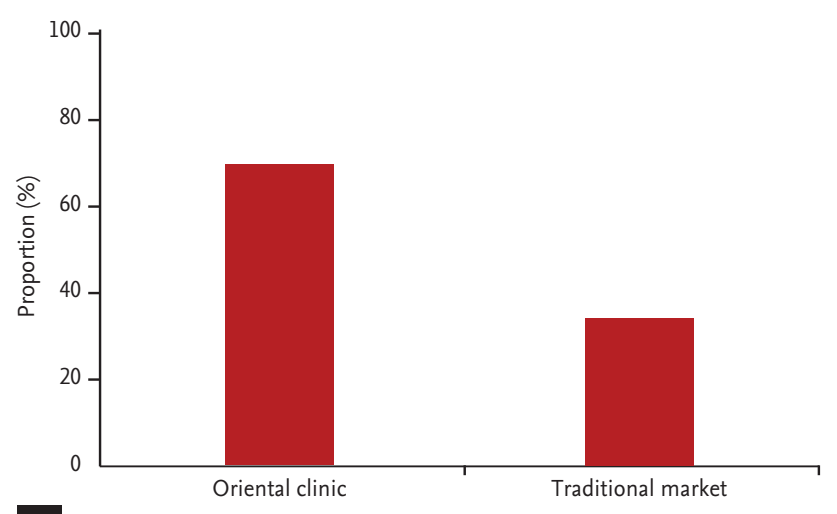

B

Figure 1. Occurrence of aristolochic acid nephropathy (AAN) and routes for obtaining herbal medicine in Korea. (A) Occurrence of AAN over a period of 20 years. (B) Routes for obtaining Chinese herbal remedies. The arrow indicates the year that Korea Food and Drug Administration prohibited AA-containing herbal medicine in Korea. 
ease (minimal change of disease in two patients, and type 2 diabetes with hypertension in one patient). Of the 16 patients, most were female $(n=14,87.5 \%)$. The occurrence of AAN based on age showed an even distribution, although the fourth and fifth decade groups were shown to be the most vulnerable (Fig. 2A). The reasons for taking oriental medicine were, in order of frequency, slimming $(\mathrm{n}=5,31.3 \%)$, control of postpartum edema and breast-feeding $(\mathrm{n}=4,25 \%)$, chronic illness $(\mathrm{n}=4$, $25 \%$ ), and health promotion ( $\mathrm{n}=3,18.8 \%)$ (Fig. $2 \mathrm{~B}$ ). These findings suggest that all age groups are exposed to the risks of AAN, and healthy females are at the greatest risk of AAN.

\section{Initial symptoms and diagnosis at hospitalization}

Fig. 3 shows the symptoms and diagnosis at initial hospitalization of all patients. Initial symptoms at admission were mostly nausea and vomiting $(\mathrm{n}=8,50 \%)$ and subsequent weakness $(\mathrm{n}=5,31.3 \%)$. Other symptoms that presented irregularly included edema, febrile sense, poor oral intake, flank pain, polydipsia, oliguria, and quadriplegia. All patients were diagnosed with AKI and had significantly elevated serum creatinine at initial hospitalization. Among them, seven patients were diagnosed with Fanconi syndrome and two patients presented with nephrotic syndrome.
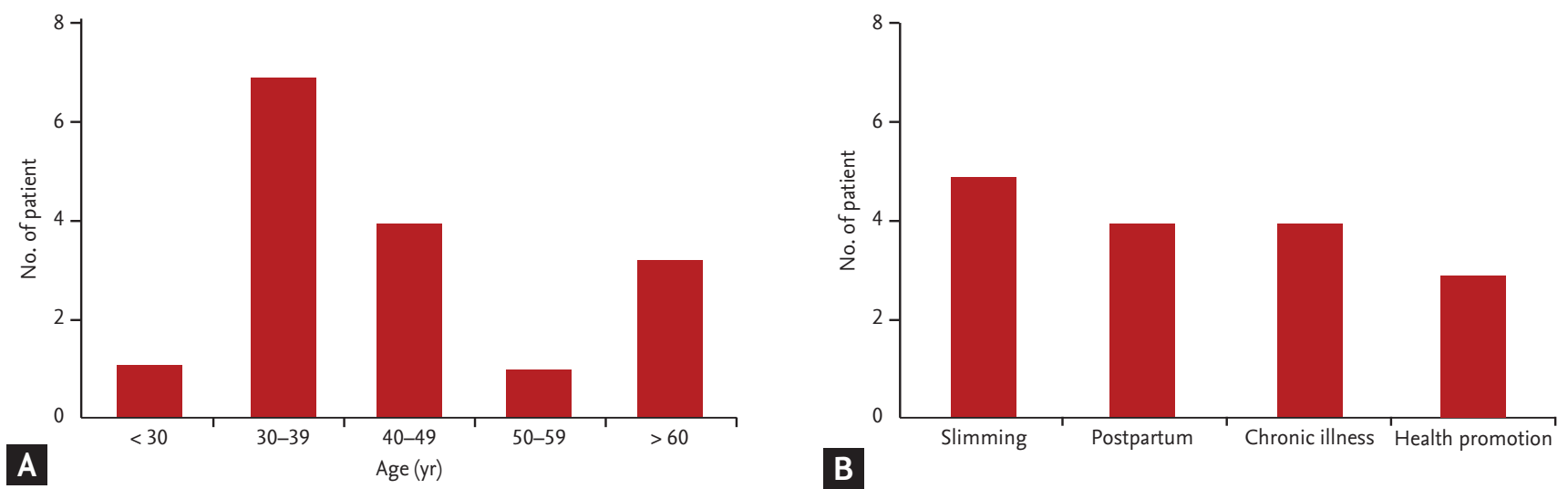

Figure 2. Age distribution of patients with aristolochic acid nephropathy (AAN) and reasons for taking herbal medicine. (A) The distribution of AAN patients in each age decade. (B) The reasons for taking Chinese herbal remedies. AAN was most commonly observed in females in the fourth or fifth decade of age.

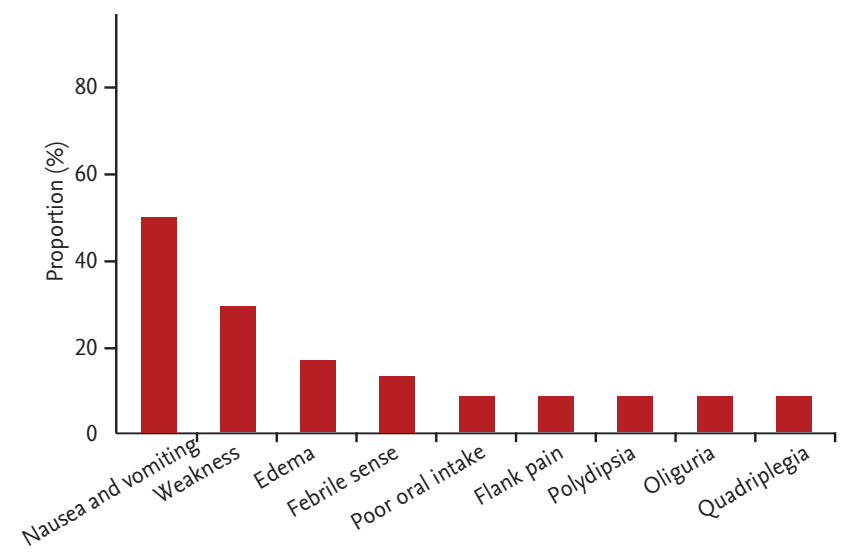

A

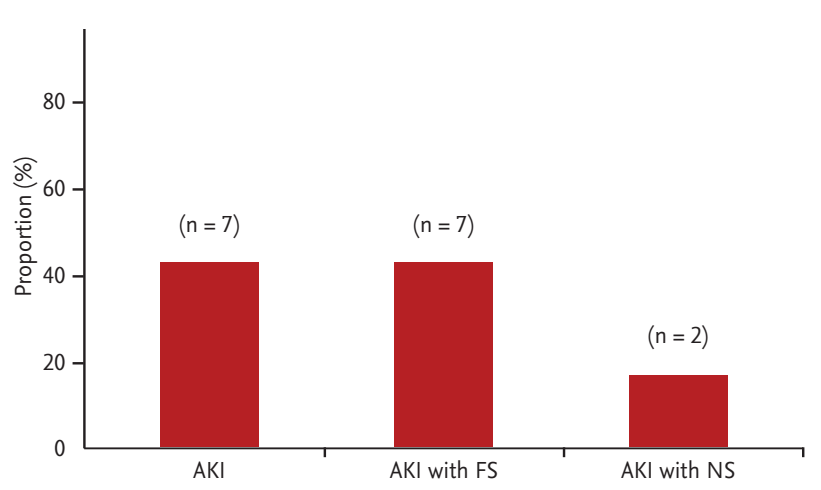

B

Figure 3. Symptoms and diagnosis of aristolochic acid nephropathy patients at initial hospitalization. (A) Initial patient symptoms. (B) Initial patient diagnosis. Note that acute gastrointestinal symptoms were the most common symptoms, and almost $50 \%$ of acute kidney injury (AKI) cases were accompanied by Fanconi syndrome (FS). NS, nephrotic syndrome 


\section{Laboratory findings of patients}

The main laboratory findings of the patients are summarized in Table 2. All patients presented with anemia and renal dysfunction, including elevated serum creatinine and proteinuria at admission. The mean value of total patients characterized the lower limit of the reference range for serum sodium, potassium, calcium, and phosphate. A larger proportion of total subjects had acidic urine $\mathrm{pH}$, glucosuria, microscopic hematuria, pyuria, and subnephrotic range proteinuria. Seven patients had Fanconi syndrome, which was diagnosed by characteristic laboratory findings such as hypokalemia, alkaline urine $\mathrm{pH}$ with metabolic acidosis, glucosuria, and aminoaciduria.

\section{Histologic findings}

The renal histopathologic findings were similar in all patients. All patients revealed characteristic findings

Table 2. Laboratory findings in patients with aristolochic acid nephropathy

\begin{tabular}{|c|c|c|}
\hline Parameter & Mean \pm SD & Range \\
\hline Hemoglobin, g/dL & $9.7 \pm 2.1$ & $6.7-12.2$ \\
\hline BUN, mg/dL & $44.7 \pm 32.2$ & $5.0-129.0$ \\
\hline Serum creatinine, $\mathrm{mg} / \mathrm{dL}$ & $5 \cdot 1 \pm 4 \cdot 4$ & $1.3-13.9$ \\
\hline $\mathrm{eGFR}^{\mathrm{a}}, \mathrm{mL} / \mathrm{min} / 1.73 \mathrm{~m}^{2}$ & $20.1 \pm 14.1$ & $2.8-47 \cdot 5$ \\
\hline Serum sodium, mEq/L & $138.4 \pm 4.6$ & $128-143$ \\
\hline Serum potassium, $\mathrm{mEq} / \mathrm{L}$ & $3 \cdot 5 \pm 1.2$ & $1.5-5 \cdot 5$ \\
\hline Serum chloride, mEq/L & $111.9 \pm 6.9$ & $105-126$ \\
\hline Uric acid, mg/dL & $5.1 \pm 3.7$ & $1.0-11.9$ \\
\hline Serum calcium, mg/dL & $8.2 \pm 0.7$ & $7 \cdot 3-9.9$ \\
\hline Serum phosphate, mg/dL & $3.6 \pm 2.0$ & $0.4^{-8.1}$ \\
\hline Total cholesterol, mg/dL & $175 \pm 71$ & $124-313$ \\
\hline Triglyceride, mg/dL & $116 \pm 62$ & $76-226$ \\
\hline LDL-C, mg/dL & $78 \pm 11$ & $62-87$ \\
\hline hs-CRP, mg/dL & $0.7 \pm 0.9$ & $0.1-1.8$ \\
\hline Urine $\mathrm{pH}$ & $5.8 \pm 0.9$ & $5 \cdot 0-7 \cdot 5$ \\
\hline Urine glucose $(+)$ & $1.7 \pm 1.6$ & $0-4$ \\
\hline Urine occult blood $(+)$ & $1.4 \pm 1.3$ & $0-3$ \\
\hline Urine leukocyte (+) & $1.2 \pm 1.3$ & $0-3$ \\
\hline Proteinuria, mg/day & $1,882 \pm 1,580$ & $426.7-6,270.0$ \\
\hline
\end{tabular}

BUN, blood urea nitrogen; eGFR, estimated glomerular filtration rate; LDL-C, low density lipoprotein cholesterol; hsCRP, high-sensitivity C-reactive protein.

${ }^{a}$ eGFR was calculated by the Modification of Diet in Renal Disease equation based on serum creatinine and patient age. such as severe tubulointerstitial nephritis, interstitial fibrosis, and tubular atrophy, whereas glomeruli were preserved in light microscopy. In addition, many cases also displayed disruption of the tubular epithelium, interstitial edema, infiltration of inflammatory cells (including lymphocytes and monocytes in the interstitium), and partial acute tubular necrosis. Fig. 4 shows the representative renal histologic findings observed by light microscopy.

\section{Concentration of AA and its clinical implication}

We obtained AA-containing remedies from six patients and measured the concentration of AA by phytochemical analysis. The concentrations of AA I, AA II, and cumulative AA I in each patient are summarized in Table 3. The mean levels of AA I and AA II were 2,070.0 mg/ $/ \mathrm{kg}$ (range, 1.9 to $8,020.0$ ) and $222.8 \mathrm{mg} / \mathrm{kg}$ (range, 1.3 to 740.0 ), respectively. The mean prescribed duration was 38.6 days (range, 10 to 120). The mean cumulative concentration of AA I was $204,898 \mathrm{mg} / \mathrm{kg}$ (range, 67.9 to 962,400 ). Of the

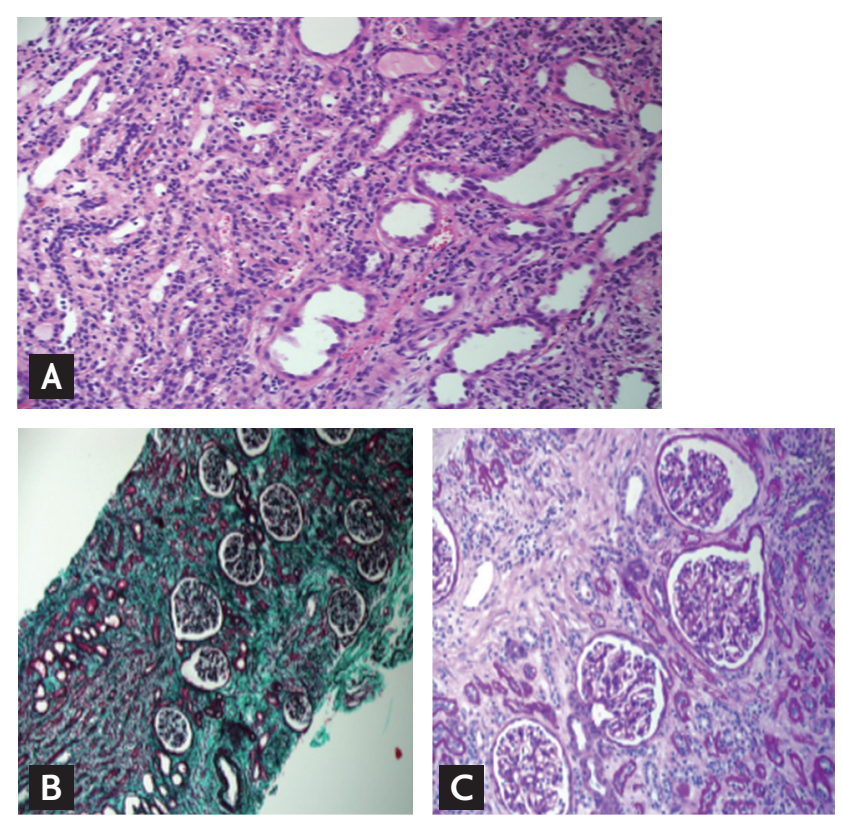

Figure 4. Representative renal histopathologic findings of aristolochic acid nephropathy (AAN). Kidney biopsy was performed in 35-year-old female patient suspected AAN. Her initial estimated glomerular filtration rate was $5.3 \mathrm{~mL} / \mathrm{min} / 1.73 \mathrm{~m}^{2}$ and she underwent emergent hemodialysis with uremic symptoms. (A) Interstitial inflammatory cell infiltration (H\&E, $\times 400)$. (B) Severe interstitial fibrosis and tubular atrophy (trichrome, $\times 400$ ). (C) Relatively preserved glomeruli (H\&E, ×400). 
Table 3. Individual data of AA level in six patients measuring AA level $(n=6)$

\begin{tabular}{|c|c|c|c|c|}
\hline Patient no. & AAI, mg/kg & AAII, mg/kg & Prescribed duration, day & Cumulative AAI, mg/kg \\
\hline $1^{a}$ & 10.1 & 1.3 & 10 & 100.6 \\
\hline $2^{a}$ & $4,357.0$ & $147 \cdot 3$ & 14 & $60,998.0$ \\
\hline 3 & 1.9 & 2.7 & UK & $\mathrm{UK}$ \\
\hline $4^{a}$ & $8,020.0$ & 740.0 & 120 & $962,400.0$ \\
\hline 5 & $4 \cdot 9$ & $\mathrm{ND}$ & 14 & 67.9 \\
\hline $6^{\mathrm{a}}$ & 26.4 & ND & 35 & 924.0 \\
\hline
\end{tabular}

Note that the amount of AA contained in herbal remedies is not consistent, and the amount of exposure to patients is diverse. AA, aristolochic acid; AAI, 8-methoxy-6-nitro-phenanthro-(3,4-d)-1,3-dioxolo-5-carboxylic acid; AAII, 6-nitro-phenanthro-(3,4-d)-1,3-dioxolo-5-carboxylic acid; UK, unknown; ND, not detected.

${ }^{a}$ Patients with progression to end stage renal disease are indicated by a star.

six cases, four with high AA I concentration and cumulative dosage showed rapid progression to ESRD within 6 months after AAN diagnosis.

\section{Clinical course of patients}

Patients showed diverse clinical courses. Progression to ESRD was observed in seven patients (43.8\%), and nine patients $(56.2 \%)$ showed progression of chronic kidney disease (CKD). The interval between AAN diagnosis and ESRD was o to 14 months, and five of seven patients (71.4\%) had progressed to ESRD within 6 months of diagnosis. Seven patients were treated with steroids at diagnosis of AAN, but it did not decrease the incidence of ESRD compared with patients who were not treated with steroids ( $14.3 \%$ vs. $44.4 \%, p=0.23$ ).

\section{DISCUSSION}

We examined the occurrence of AAN in Korea during the past 20 years. The results of our study show that the outbreak of AAN decreased after the ban of AA-containing ingredients by the KFDA in 2005, but AAN still occurred sporadically after the KDFA prohibition. AA-containing herbal medicine was available through official (oriental medicine clinics) and non-official routes (traditional drug markets). The results of our study revealed that the risk of AAN is still present in Korea even after the legal prohibition of AA-containing herbal medicine, and people are still exposed to AA-containing herbal compounds.

The reason for the high occurrence of AAN in young females is that herbal medicine is thought to be an effec- tive treatment for weight control without complications [23]. Therefore, most reports of AAN are related to herbal remedies used for slimming purposes. Furthermore, AAN due to herbal medicine is also known to occur in a wide variety of remedies for diseases or poor health conditions such as eczema, hepatitis B, liver function enhancement, arthritis, rheumatism, and pain relief $[1,2]$. The use of Chinese herbal medication to improve health is popular in Korea. For this reason, herbal medicine is favored in a wide range of age groups, as observed in this study (Fig. 2A). Compared to reports in other countries, AAN in Korea was characterized by a high occurrence in females during the postpartum period. The main purpose of herbal medicine in this group was for alleviation of post-partum edema and to improve breast-feeding. Therefore, the possibility that AA exposure to mothers can be transferred to babies via breast feeding should be taken into consideration, and further studies are needed on this point.

Reported cases of AAN were usually asymptomatic on initial presentation, and renal failure was often discovered by routine blood testing $[1,23]$. Therefore, it is difficult to diagnose AAN if clinicians do not consider the association between AKI and herbal medicine. In our study, we identified some clues to clinically suspect AAN. First, the most important symptoms to suspect AAN were gastrointestinal (GI) symptoms such as nausea and vomiting. Many patients stopped taking herbal medicine due to these symptoms. Second, laboratory findings of Fanconi syndrome were helpful in suspecting AAN. In our study, seven patients showed both AKI and Fanconi syndrome, which is quite unusual in young people. Finally, renal biopsy findings of interstitial ne- 
phritis were strongly suggestive of AAN. In our study, pathologists strongly suggested the diagnosis of AAN based on characteristic histologic findings. Therefore, we suggest that clinicians should suspect AAN in patients presenting with acute GI symptoms, unexplained AKI, and/or Fanconi syndrome with interstitial nephritis on kidney biopsy.

In our study, only $37.5 \%$ ( 6 out of 16 cases) of the cases were able to undergo phytochemical analyses. This small percentage can be explained in two ways. First, many individuals discard the remainder of their herbal medicine once complications arise. Therefore, in many cases, we can suspect AAN but are unable to make a definite diagnosis. Second, measurement of AA in herbal medicine is not available in clinical practice because of technical difficulties and high costs for measuring the concentration of AA. Indeed, in our study, phytochemical analysis was performed at the researcher's laboratory or at a public toxicology laboratory. Based on phytochemical analysis, we found that the amount of AA contained in herbal remedies is not consistent (ranging from 4.9 to 43,570 $\mathrm{mg} / \mathrm{kg}$ ), and the mean concentration of AA was higher than the dose used for developing acute AAN in experimental animal models [1]. This can explain the wide range of renal dysfunction and the high proportion of acute GI symptoms in patients at admission.

The exact mechanisms of AAN have not been fully understood. As reported in several in vivo and in vitro studies, the proximal tubule is the target of AA. Atrophy and apoptosis is involved in the progression of AA-induced proximal tubular denudation $[1,24]$. In clinical studies, cumulative AA dose is the only risk factor for the development and progression of renal disease. The Belgian cohort showed that the ingested dose of emerged Aristolochia fangchi was strongly associated with the progression of renal failure [25], and retrospective cohort and case control studies carried out in China and Taiwan have reported median doses of AA associated with renal impairment $[26,27]$. We also calculated the cumulative doses of AA and found that patients who progressed to ESRD received higher cumulative doses than patients who received lower doses. Taken together, results show that the cumulative dose of AA is a risk factor for the progression of renal failure, and the formation of AADNA adducts potentially leads to atrophy and apoptosis in proximal tubules [24].
In terms of clinical outcomes in our study, seven patients $(43.8 \%)$ showed progression to ESRD, and nine patients (56.2\%) had ongoing CKD during the follow-up period. This finding was consistent with previous reports showing that AAN has variant phenotypes with distinct prognosis. In AAN patients with progression to $\mathrm{CKD}$, steroid therapy is recommended for patients with an estimated glomerular filtration rate greater than 20 $\mathrm{mL} / \mathrm{min} / 1.73 \mathrm{~m}^{2}[6,23]$, and its use is extended to patients with advanced renal dysfunction [28]. In our study, we administered steroid treatment to seven patients, but this did not significantly delay progression to ERSD compared with non-treated patients. However, because our study included a small number of AAN patients with varying degrees of renal dysfunction, this result does not imply that steroid treatment is not effective in delaying the progression of AAN. Another important factor is the presence of Fanconi syndrome. Previous reports have suggested that the presence of Fanconi syndrome predicts favorable clinical outcomes [26]; however, our study showed that $42.9 \%$ of AAN patients with Fanconi syndrome progressed to ESRD. This finding suggests that the presence of Fanconi syndrome does not indicate a benign clinical course, and we recommend more frequent follow-up of renal function in AAN patients with Fanconi syndrome.

There are several reasons for the persistent occurrence of AAN after KFDA prohibition of AA in Korea. First, AA-containing compounds were not properly screened. It is strongly suspected that Aristolochia spp. plants were mistakenly identified as Tetrapanax papyriferus and Stephania tetrandra because of similarities in their appearance. Second, Chinese medicines can be easily acquired in traditional markets and on the Internet $[1,16]$, since there is not a clear distribution channel for herbal medicine. Third, oriental medicine in Korea lacks scientific evidence on its safety and effectiveness. The use of herbal remedies is based on knowledge obtained through historic medical texts written in the 17th century. Therefore, the safety and effectiveness of herbal medicine are still unknown. Furthermore, there is a risk of outbreak of AAN because several oriental clinics function as dispensary centers for Chinese medicine. Unfortunately, it is quite difficult to avoid AAN under the current screening system and distribution route, and it might be necessary to perform toxicity tests before the ingredients of 
traditional medicine are distributed to clinics in order to prevent AAN.

Our study has some limitations. First, this study is retrospective. Second, the included number was too small to evaluate the clinical outcome. Third, only six cases were available to measure AA levels in remained herbal medicine. Fourth, data on baseline renal function were not available. Fifth, there is a selection bias since we included only four hospitals. We expect that more cases may be included if a national survey is conducted. In spite of these limitations, this report suggests that there is a possibility of AAN among patients previously diagnosed as having chronic tubulointerstitial nephritis of unknown origin, and more patients will be included if clinicians pay attention to the possibility of AAN.

In conclusion, $\mathrm{AA}$ is one of the most important causes of renal tubular damage and interstitial nephritis leading to progressive kidney injury. We believe that AAN is a preventable cause of ESRD via a strong national supervising system for AA-containing herbal medicine in oriental clinics and traditional markets.

\section{KEY MESSAGE}

1. Aristolochic acid (AA)-containing herbal medicine is still available via various routes.

2. Because the definite incidence of aristolochic acid nephropathy (AAN) is unknown and there is a possibility of underdiagnosis, clinicians should suspect AAN in patients presenting with acute gastrointestinal symptoms, unexplained acute kidney injury, and/or Fanconi syndrome with interstitial nephritis.

3. A stronger national supervising system on herbal ingredients and remedies in oriental medicine is needed to prevent AAN.

\section{Conflict of interest}

No potential conflict of interest relevant to this article was reported.

\section{Acknowledgments}

This study was supported by a grant of the Korean Health Technology R\&D Project, Ministry for Health and Welfare, Republic of Korea (HI14C3417).

\section{REFERENCES}

1. Debelle FD, Vanherweghem JL, Nortier JL. Aristolochic acid nephropathy: a worldwide problem. Kidney Int 2008;74:158-169.

2. Krell D, Stebbing J. Aristolochia: the malignant truth. Lancet Oncol 2013;14:25-26.

3. Vanherweghem JL, Depierreux M, Tielemans C, et al. Rapidly progressive interstitial renal fibrosis in young women: association with slimming regimen including Chinese herbs. Lancet 1993;341:387-391.

4. Nortier JL, Martinez MC, Schmeiser HH, et al. Urothelial carcinoma associated with the use of a Chinese herb (Aristolochia fangchi). N Engl J Med 2000;342:1686-1692.

5. Arlt VM, Stiborova M, Schmeiser HH. Aristolochic acid as a probable human cancer hazard in herbal remedies: a review. Mutagenesis 2002;17:265-277.

6. Gokmen MR, Cosyns JP, Arlt VM, et al. The epidemiology, diagnosis, and management of aristolochic acid nephropathy: a narrative review. Ann Intern Med 2013;158:469-477.

7. Pena JM, Borras M, Ramos J, Montoliu J. Rapidly progressive interstitial renal fibrosis due to a chronic intake of a herb (Aristolochia pistolochia) infusion. Nephrol Dial Transplant 1996;11:1359-1360.

8. Lord GM, Tagore R, Cook T, Gower P, Pusey CD. Nephropathy caused by Chinese herbs in the UK. Lancet 1999;354:481-482.

9. Tanaka A, Nishida R, Maeda K, Sugawara A, Kuwahara T. Chinese herb nephropathy in Japan presents adult-onset Fanconi syndrome: could different components of aristolochic acids cause a different type of Chinese herb nephropathy? Clin Nephrol 2000;53:301-306.

10. Yang CS, Lin CH, Chang SH, Hsu HC. Rapidly progressive fibrosing interstitial nephritis associated with Chinese herbal drugs. Am J Kidney Dis 2000;35:313-318.

11. Lee $\mathrm{S}$, Lee T, Lee B, et al. Fanconi's syndrome and subsequent progressive renal failure caused by a Chinese herb containing aristolochic acid. Nephrology (Carlton) 2004;9:126-129.

12. Laing C, Hamour S, Sheaff M, Miller R, Woolfson R. Chinese herbal uropathy and nephropathy. Lancet 2006;368:338.

13. Chau W, Ross R, Li JY, Yong TY, Klebe S, Barbara JA. Nephropathy associated with use of a Chinese herbal product containing aristolochic acid. Med J Aust 2011;194:367368.

14. Hutton H, Dowling J, Kerr P. Acute renal failure due to an 
aristolochic acid containing herbal remedy. Nephrology (Carlton) 2012;17:522-523.

15. Tazi I, Nafil H, Mahmal L. Fatal renal failure due to self administration of Aristolochia Longa after treatment with chemotherapy. Arab J Nephrol Transplant 2012;5:54.

16. Vaclavik L, Krynitsky AJ, Rader JI. Quantification of aristolochic acids I and II in herbal dietary supplements by ultra-high-performance liquid chromatography-multistage fragmentation mass spectrometry. Food Addit Contam Part A Chem Anal Control Expo Risk Assess 2014;31:784-791.

17. Jeong HJ, Choi KH, Park JH, et al. Nephropathies associated with herb medicine ingestion. Kidney Res Clin Pract 1996;15:365-372.

18. Nam KD, Lee TW, Noh JH, et al. A case of Chinese herbs nephropathy. Korean J Nephrol 2000;19:751-755.

19. Choi CR, Yun YW, Lee DK, et al. A case of Fanconi's syndrome due to Chinese herb nephropathy: differences between European and Asian CHN. Korean J Nephrol 2003;22:118-123.

20. Lee JW, Sohn MJ, Heo NJ, et al. A case of Chinese herb nephropathy induced by Aristolochia fangchi. Korean J Med 2006;71:224-228.

21. Sung HY, Shin SJ, Son SW, et al. Chinese Herbs induced end-stage renal disease in a patient with minimal change nephrotic syndrome. Korean J Nephrol 2006;25:99-102.

22. Won HS, Cho IJ, Yoo SH, et al. A case of Fanconi's syndrome due to Chinese herb nephropathy. Ewha Med J 2007;30:101-105.

23. Luciano RL, Perazella MA. Aristolochic acid nephropathy: epidemiology, clinical presentation, and treatment. Drug Saf 2015;38:55-64.

24. Pozdzik AA, Salmon IJ, Debelle FD, et al. Aristolochic acid induces proximal tubule apoptosis and epithelial to mesenchymal transformation. Kidney Int 2008;73:595-607.

25. Martinez MC, Nortier J, Vereerstraeten P, Vanherweghem JL. Progression rate of Chinese herb nephropathy: impact of Aristolochia fangchi ingested dose. Nephrol Dial Transplant 2002;17:408-412.

26. Yang L, Su T, Li XM, et al. Aristolochic acid nephropathy: variation in presentation and prognosis. Nephrol Dial Transplant 2012;27:292-298.

27. Chen CH, Dickman KG, Huang CY, et al. Aristolochic acid-induced upper tract urothelial carcinoma in Taiwan: clinical characteristics and outcomes. Int J Cancer 2013;133:14-20.

28. Martinez MC, Nortier J, Vereerstraeten P, Vanherweghem JL. Steroid therapy in chronic interstitial renal fibrosis: the case of Chinese-herb nephropathy. Nephrol Dial Transplant 2002;17:2033-2034. 